$\underline{\text { Review }}$

\title{
Salmonella Typhimurium as a potential anticancer agent: A Review
}

\author{
SO Enejiyon ${ }^{1}$, NU Adabara ${ }^{1}$, MM Wuna $^{1}$, RA Fasasi $^{1}$ \\ Sri Lankan Journal of Infectious Diseases 2020 Vol.10(2):98-113 \\ DOI: http://dx.doi.org/10.4038/sljid.v10i2.8289
}

\begin{abstract}
Bacteria mediated cancer therapy has not been used as much as other methods, despite being considered a potential adjunct in anticancer therapeutic strategy for decades. However, in recent years, there has been considerable interest in exploration of the option of bacteria mediated cancer immunotherapy. The conventional anticancer therapy does not eradicate cancer completely. It often fails and has several other limitations which can easily be overcome through the bacteria mediated approach as an adjunctive therapy.
\end{abstract}

Members of the genus Salmonella have the ability to colonize all forms of tumours and their metastasis much more efficiently than other bacteria. Salmonella has over 2500 serovars of which Salmonella Typhimurium, a non-typhoidal strain, is the most extensively studied for its anticancer activity. $S$. Typhimurium has the intrinsic attribute of being able to selectively colonize solid tumours and their metastasis.

$S$. Typhimurium is able to target and destroy tumours in three specific ways; inducing immune response to the presence of tumours, utilizing bacterial toxins to directly activate caspase-3, ( an important enzyme of the apoptotic pathway) and also as a vector in delivering of anti-cancer compounds to tumour sites. $S$. Typhimurium is currently considered as a bacterium with great potential in the field of cancer immunotherapy. In this review, the explanation of the mechanisms of anticancer activity of live attenuated and engineered $S$. Typhimurium strains in vitro and in vivo is attempted.

Keywords: bacteria, cancer, immunotherapy, Salmonella, tumours.

\section{Introduction}

Cancer is a major cause of death in millions of individuals throughout the world. ${ }^{1}$ According to the global observatory report of the International Agency for Research on Cancer (IARC), the

${ }^{1}$ Department of Microbiology, School of Life Sciences, Federal University of Technology Minna, Nigeria Address for correspondence: SO Enejiyon, Department of Microbiology, School of Life Sciences, Federal University of Technology Minna, Nigeria. Tel: +2348161320129

E-mail: enejiyon.sherifat@futminna.edu.ng (1D https://orcid.org/0000-0002-7016-6001

Received 4 April 2020 and revised version accepted 8 July 2020

C) (7)

This an open-access article distributed under the terms of the Creative Commons Attribution License, which permits unrestricted use, distribution, and reproduction in any medium, provided the original author and source are credited. 
burden of cancer in 2018 is estimated to have risen to about 18.1 million new cases and 9.6 million deaths. One in 5 men and one in 6 women will develop cancer during their lifetime before the age of 75 and one in 8 men and one in 11 women will die from the disease. ${ }^{2}$ Cancer is a leading cause of economic loss worldwide due to premature deaths, disabilities, vast sum of money spent on treatment and also the loss of economic and social activity. The World Health Organization (WHO) estimated that the number of cancer cases will increase to 19 million by 2025, 22 million by 2030 and 24 million by 2035 . More than $60 \%$ of the global cases of cancer occur in Africa, Asia and Central and South America, and these regions also tend to account for about $70 \%$ of the world cancer deaths. ${ }^{2}$

\section{Oncotherapy}

Among the well-known and practiced strategies used in oncotherapy, surgery is the most common conventional cancer treatment approach and has been used for decades. However, it has not been an effective treatment for metastatic disease which requires combination treatment involving other conventional therapies such as radiation and chemotherapy. ${ }^{3}$ Conventional therapies are characterized by poor survival rates due to several limitations. Surgery and radiation therapy are associated with being limited to localized tumours. ${ }^{4}$ Chemotherapy may induce severe side effects including, toxicity, poor tumour targeting, limited tissue penetration, development of drugresistance and lack of tumour specificity, resulting in undesirable side effects on healthy cells. ${ }^{5}$ These limitations are infrequently associated with incomplete eradication of cancerous cells, possible regrowth and/or secondary neoplasia. ${ }^{6}$ It is extremely important to find effective adjuncts that can complement existing treatment regimens in tackling the menace of cancer that has remained one of the major challenges of the 21 st century. Immunotherapy using bacteria happens to be a promising research direction that needs to be fully explored. ${ }^{7}$

\section{Bacteria-mediated oncotherapy}

The idea of treating tumours with bacteria was first explored in the middle of the $19^{\text {th }}$ century when Dr. William Coley (1862-1936) observed the regression of tumours in patients who developed postoperative bacterial infections. ${ }^{8}$ This observation prompted him to develop a mixture of bacterial extracts (Streptococcus pyogenes and Serratia marcescens) to successfully treat patients with different types of cancer. Bacteria were previously considered as one of the primary agents involved in the causation of cancer. However, recent research has shown that bacteria can be an effective agent for cancer treatment. ${ }^{7}$ Bacteria-mediated oncotherapy has evolved to overcome most of the limitations associated with conventional therapeutic methods. Bacterial species can also be manipulated genetically to overcome many of the limitations that frequently impede cancer therapy by directly targeting cancer cells, destroying these cells through innate bacterial toxicity, competing for nutrients or acting as a vector in delivery of anti-cancer agents. ${ }^{9}$

The use of bacteria for cancer therapy has many advantages over conventional cancer therapies. The amount of bacteria accumulation in tumours is approximately one thousand times higher than in normal organs. ${ }^{10}$ The highly proliferative nature of the bacterium enhances its therapeutic effect, thereby circumventing the need for external supply. Although these bacteria grow in viable as well as necrotic regions of tumours, nutritional auxotrophy severely limits growth in normal tissues.

Several species and strains of anaerobes (obligate and facultative) have been explored for their possible anti-cancer potential. These include Mycobacterium bovis, Streptococcus pyogenes OK 
432, Serratia marcescens, Magnetococcus marinus, Clostridium sp., Bifidobacterium sp., Listeria sp., Escherichia coli and Salmonella sp. Among all these bacteria, S. Typhimurium has been the most extensively studied for its potential use in cancer therapy with varying degrees of success. ${ }^{11-}$ 15

S. Typhimurium, a non-typhoidal Salmonella strain which is a Gram-negative, facultative anaerobic bacterium, is able to grow and replicate within the cells of it host. ${ }^{16} S$. Typhimurium can penetrate deep into tumours and are unaffected by the tumour's immune evasion strategies. In addition, $S$. Typhimurium is motile and can easily be manipulated genetically.

A large number of studies have shown the immunotherapeutic role of $S$. Typhimurium strain both in vitro and in murine models. ${ }^{17,18}$ Attenuated and engineered $S$. Typhimurium strains have been demonstrated to eliminate virulence while provoking anti-tumour activity through different mechanisms. ${ }^{19} S$. Typhimurium also serves as a suitable vector in antitumour immunotherapy ${ }^{15}$ with the following characteristics; their tumour colonizing ability ${ }^{20,21}$, metastasis ${ }^{12}$ and affinity for antigen-presenting cells. ${ }^{22}$ Systemic infection with Salmonella stimulates the expression of proinflammatory cytokine and infiltration of immune cell in the host due to immunostimulation by Salmonella lipopolysaccharide and other components. ${ }^{23}$

\section{Selectivity of $S$. Typhimurium for tumour tissue}

The choice of $S$. Typhimurium as a therapeutic alternative against cancer, ${ }^{11,24}$ is due mainly to the ability of the bacterium to selectively colonize the tumour microenvironment. ${ }^{25} S$. Typhimurium is able to successfully penetrate and invade tumour tissue as they are drawn towards chemical compounds produced by cancer cells such as aspartate, serine, citrate, ribose or galactose, which serve as chemoattractants and preferentially grow within tumour tissues. ${ }^{14}$ Migration of the organism towards the tumour site is based on the ability of the organism to sense nutrients using receptors that are located on the outer membrane of the bacterial cell. ${ }^{26}$ Two of those receptors have been characterized; the TAR receptor (which detects aspartate secreted by cancerous tissues) and the TRG receptor (which detects ribose found in necrotic tissues). Ethanolamine, a chemical compound found in high concentration in neoplasia ${ }^{27}$ has also been shown to act as a chemotactic agent. The excision of the eutC gene (portion of the operon encoding the enzyme ethanolamineammonia-lyase which metabolizes ethanolamine) in $S$. Typhimurium, reduced its colonization in a murine model of breast cancer. ${ }^{28,29}$

The tumour microenvironment is characterized by hypoxia, with oxygen concentrations ranging between 10 to $30 \mathrm{mmHg}$ which is caused by rapidly growing tumours with insufficient blood supply, ${ }^{15,30}$ acidity that is conditioned by lactic acid resulting from anaerobic metabolism due to reduced oxygen, ${ }^{31}$ and necrosis resulting from the death of tumour cells due to insufficient nutrients and excessive uncontrolled growth. ${ }^{24}$ These characteristics are thought to explain why $S$. Typhimurium selectively colonizes tumour tissues. The anaerobic property of hypoxic or necrotic regions within tumours enhances growth of both obligate and facultative anaerobes. ${ }^{24}$ In addition, areas of necrosis may also supply nutrients including purines to further promote the growth of bacteria in the tumour. ${ }^{32}$ 


\section{Direct tumour-killing activity of $S$. Typhimurium}

The mechanisms underlying the direct anti-tumour activities of Salmonella have not been clearly elucidated but it is recognized that several mechanisms are involved in the Salmonella-induced killing of tumour cells. ${ }^{33}$ Multiple mechanisms are utilized by $S$. Typhimurium to induce tumour apoptosis and this includes, but is not limited to competition for nutrients, stimulation of immune response and production of toxins. ${ }^{23}$

Salmonella may also cause death of tumour cells directly by the activation of apoptosis and autophagic pathways using 3-methyladenine (an autophagy inhibitor) and Z-VAD-FMK (an apoptosis inhibitor). ${ }^{34}$ The signalling pathways leading to the activation of autophagy induced by bacteria in tumour cells is still being investigated. One of the pathways found to negatively regulate autophagy in tumour cells is the AKT/mTOR/p70S6K signalling pathway. ${ }^{34,35}$ Levels of phosphorylated AKT, mTOR, and p70S6K decreased significantly in tumour cells treated with $S$. Typhimurium. ${ }^{36}$ These results showed that Salmonella can induce autophagic activities as well as caspase-dependent apoptosis in tumour cells. Autophagy may occur simultaneously with apoptosis in tumour cells exposed to Salmonella. Also, at later phases of infection, autophagy may partially participate in death of tumour cells by enhancing apoptosis. When apoptosis is barred, infected tumour cells undergo enhanced autophagy. Salmonella treatment efficiently destroys tumour cells by inducing both autophagy and apoptosis, which combine to induce cell death cooperatively by modifying the expression of beclin-1 and caspase. ${ }^{19}$

Bacterial replication within tumours and eventual lysis of tumour cells may stimulate cellmediated immune responses to tumour cells. Higher oncolysis could account for an increased infiltration of immune cells into tumours. The cells undergoing Salmonella-induced cell death show heterogeneous morphological characteristics. ${ }^{37}$ 


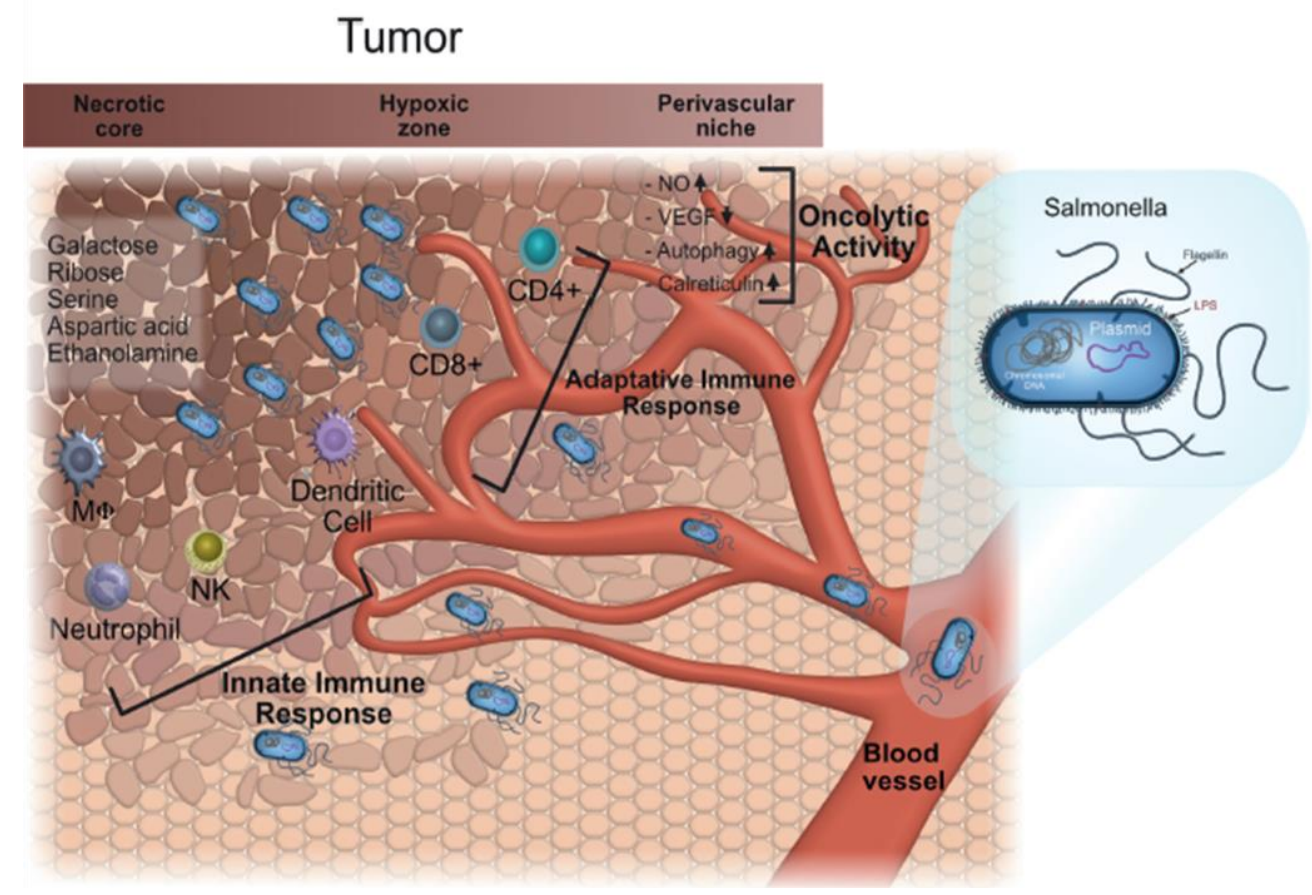

Figure 1: $S$. Typhimurium selectivity for the tumour, oncolytic activity and activation of immune response

Source: Hernández-Luna MA, Munoz-Lopez P, Luria-Perez R, et al. Infection by Salmonella enterica promotes or demotes tumour development. Salmonella - a reemerging pathogen 2018; 54-71 doi: https:// doi.org/10.5772/intechopen.75481

S. Typhimurium is attracted to the tumour site by chemoattractants such as galactose, ribose, serine, aspartic acid and ethanolamine. Presence of $S$. Typhimurium in tumour microenvironment leads to recruitment of phagocytic cells, secretion of cytokines, activation of CD4+ and CD8+ and expression of soluble mediators such as nitric oxide (NO) and VEGF which are essential for activation of immune response and direct oncolytic activity.

\section{Activation of host immune system by Salmonella-tumour interaction}

Invasion of $S$. Typhimurium into the body is expected to stimulate the production of specific immune reactions against specific types of cancer cells. ${ }^{38,39}$ Two possible mechanisms have been proposed to explain the role of Salmonella in activating the host immune system; the chemotactic and motility-based system ${ }^{12}$ and the passive initiation of secretion of infiltrating pro-inflammatory cytokines upon intravenous injection of Salmonella. ${ }^{32}$

The development of several immune evading strategies by tumour cells have enabled the generation of more aggressive phenotypes, which have resulted in the development of resistance to tumour immune therapy. ${ }^{40}$ The immune response against Salmonella is composed of an immediate response mediated by the innate immune system followed by adaptive immune system. ${ }^{41}$ The host immune system also plays a significant role in the regulation of Salmonellatumour interaction in vivo. 


\section{Activation of innate immunity by Salmonella}

$S$. Typhimurium can promote anti-tumour immunity as a potent natural adjuvant by activating innate immune cells followed by secretion of cytokines that can recruit and activate other immune cells at the tumour site. ${ }^{42}$ In the tumour microenvironment, $S$. Typhimurium induces the reversal of the suppressor environment by enabling the expression of soluble mediators, including inducible nitric oxide synthase and interferon- $\gamma$ molecules that promote antitumour activity and inhibition of immunosuppressive factors such as interleukin-4 (IL-4) and arginase-1, transforming growth factor-beta (TGF- $\beta$ ) and vascular endothelial growth factor (VEGF). ${ }^{11,38} S$. Typhimurium expresses soluble mediators which may play a significant role in decreasing the activity of myeloid-derived suppressor cells ${ }^{43}$ and promoting the recruitment of intra-tumoural natural killer cells (NK), ${ }^{44}$ neutrophils, ${ }^{45}$ macrophages, ${ }^{38}$ T lymphocytes ${ }^{41}$ and B lymphocytes. ${ }^{46}$

It was previously reported that lipopolysaccharide (LPS) found in $S$. Typhimurium might be crucial in the activation and recruitment of immune cells and production of tumour necrosis factoralpha (TNF- $\alpha$ ). TLR4 signalling is involved in the Salmonella-induced cytokine expression. ${ }^{47}$

Anti-tumour effectiveness of Salmonella depends greatly on the induction of the innate immune response through the toll-like receptor-myeloid differentiation primary response gene (TLRMYD88) signalling pathway. ${ }^{38}$ This is similar to an earlier report that Salmonella induces cytokine production and antitumour activities through toll-like receptor 4 (TLR4) signalling, which may aid the clarification of the molecular mechanism of Salmonella-induced host antitumour responses. ${ }^{48}$ Increased expression of interferon-induced chemokines in the tumour was observed during Salmonella treatment in vivo. Interferon-dependent chemokines induced by Salmonella, such as monokines induced by Interferon- $\gamma$ (MIC) and IFN-inducible protein-10 (IP10), are expected to recruit activated effector cells within the tumour. It was further reported that increased expression of pro-inflammatory cytokines (IL-1b and TNF- $\alpha$ ) by $S$. Typhimurium into the tumour environment results in tumour regression in response to $S$. Typhimurium infection. ${ }^{49}$ In contrast, TNF- $\alpha$ causes apoptosis-induced tumour cell death, IL-1b causes CD8+ cytotoxic T lymphocytes and CD4+ T cells induce tumour cell death. The increased TNF- $\alpha$ in the tumour microenvironment would therefore promote bleeding from the blood vessels of the tumour and allow infiltration by immune response cells which leads to elimination of the tumour cells. ${ }^{50}$ Additionally, the presence of $S$. Typhimurium in tumour tissues increases the number of immune effector cells in the spleen, which subsequently migrates to the tumour and contribute to its eradication. $^{51}$

\section{Activation of anti-tumour adaptive immunity by Salmonella}

Salmonella-induced immune responses, especially the adaptive immune response, may include both anti-Salmonella-specific and antitumour-specific antigen responses. ${ }^{52,53} \mathrm{~S}$. Typhimurium has been utilized as a delivery system of tumour-associated antigen or tumour-specific antigen. ${ }^{54}$ Expression and release of tumour-associated antigen or tumour-specific antigen through type I and type III secretion systems of $S$. Typhimurium has the role of inducing a specific immune response against the tumour, considering the great tropism of $S$. Typhimurium for antigen-presenting cells. ${ }^{55}$

Salmonella can cause formation of gap junctions (Cx43) between dendritic cells of the immune system and melanoma cells. ${ }^{56}$ The dendritic cells employ peptides transferred from the tumour 
cells to induce $\mathrm{T}$ cells to recognize and kill tumour cells and also prevent metastasis formation. ${ }^{53}$ This process has been observed in solid tumours, their metastases,${ }^{57}$ as well as, nonsolid tumours. ${ }^{44}$ Salmonella act by recruiting $\mathrm{T}$ cells that subdue tumour growth and systemically encourage development of the immune response via the cross-presentation of tumour antigen. ${ }^{53}$

$S$. Typhimurium and tumour interaction upregulation of the cellular protein connexin 43 also results in gap junction formation between cancer cells and antigen presenting cells, thus allowing antigen presenting cells access to pre-processed tumour antigens, which they can then present to T-cells. ${ }^{58}$ Lysis of tumour cells by Salmonella may cause cell-mediated immune responses to tumour cells by increasing the infiltration of $\mathrm{CD}^{+} \mathrm{T}$ cells in the tumour cells. The cytotoxic $\mathrm{T}$ cell response against tumour cells may increase the antitumour efficacy of cytokines showing an ability to regulate host immunity and suppress tumour growth recruited by Salmonella ${ }^{47,56,59}$ Following injection of Salmonella into the host cell, strong host immunity may develop, and also, synthesis of specific antibodies directed against Salmonella. The immune responses against Salmonella existing within the host influence the tumour-targeting potential of Salmonella after systemic administration. The limited accumulation of Salmonella within the tumour contributes to the inhibition of the Salmonella-mediated antitumour response. These results may explain the limited accumulation of Salmonella within tumour sites and the non-significant antitumour response after systemic administration in clinical trials. ${ }^{60}$

\section{Engineered $S$. Typhimurium}

$S$. Typhimurium mediated cancer immunotherapy has some limitations which can be countered by the use of engineered or attenuated strains. Some of the limitations include the trigger of host antibacterial responses upon an increase in the concentration of bacteria, which ultimately lead to clearance of the introduced bacteria. ${ }^{61}$

Prior exposure to the organism may hinder the therapeutic potential of bacteria-mediated tumour therapy and the accompanied cytotoxic effect. ${ }^{60,62}$ This limiting effect of prior exposure was addressed by engineering Salmonella Typhimurium strain SF200, resulting in a modified Lipid A structure through the deletion of DlpxR9, DpagL7 and DpagP8 genes, and mutations of DydiV and DfliF genes to modify flagella synthesis. ${ }^{62}$

Various strategies have been employed to engineer the bacterium in order to reduce its cytotoxic effects on normal cells. The cytotoxic effect is a major limitation in the development of a safe Salmonella strain for immunotherapy. This effect can be reduced by incorporating certain genetic manipulations in the organism to enhance its efficiency in tumour regression. ${ }^{63}$ A Salmonella strain with a synchronized lysis circuit, mediated by a quorum-sensing lysis system, which permits the release of toxic substances in a wobbling manner into the tumour was developed. ${ }^{64}$ This strain caused a notable reduction of tumour activity along with reduced cytotoxicity on normal cells.

A strategy for controlling therapeutic agent delivery is the engineering of Salmonella to produce cytosine deaminase; an enzyme that cleaves the pro-drug 5-fluorocytosine to the active chemotherapeutic agent (5-fluorouricil) inside the tumour. ${ }^{65,66}$ A genetically engineered strain of S. Typhimurium (A1-R) was able to specifically target and penetrate cancer cells. ${ }^{57}$ As monotherapy, $S$. Typhimurium A1-R was able to inhibit or eliminate primary and metastatic tumours in mouse models of prostate, ${ }^{67}$ breast ${ }^{68}$ lung, ${ }^{69}$ pancreas, ${ }^{70,71}$ ovaries,${ }^{72}$ stomach, ${ }^{73}$ 
cervix, ${ }^{74}$ sarcoma $^{75}$ and glioma, ${ }^{76,77}$ all of which are extremely aggressive tumours. Some genetically manipulated strains of $S$. Typhimurium that have been developed for various studies and clinical trials in cancer immunotherapy are presented in Table 1.

\section{Table 1: Genetically Engineered Salmonella Typhimurium strains}

\begin{tabular}{|c|c|c|}
\hline Strain & Mutation & Tumour \\
\hline S14028 & Pur, ilv, arg, ura, aro & Melanoma $^{21}$ \\
\hline SL7207 & $\begin{array}{l}\text { aroA, hisG46, cheY, fliGHI, } \\
\text { invG, phoP, sseD }\end{array}$ & Colon cancer ${ }^{78}$ \\
\hline SL1344 & ssrB, purA & Colon cancer ${ }^{78}$ \\
\hline SL3235 & aroA & Plasmocytoma $^{79}$ \\
\hline VNP20009 & purI, msbB & Metastasic melanoma ${ }^{80}$ \\
\hline Wild-type LT2 & $\begin{array}{l}\Delta \text { ppGpp, hisD2550, rpoS, } \\
\text { aroA, rfaH, thyA }\end{array}$ & Prostate cancer, Breast cancer ${ }^{81}$ \\
\hline $\begin{array}{l}14028 \text { auxotrophy } \\
\text { A1 and A1-R }\end{array}$ & Leu, Arg & $\begin{array}{l}\text { Breast cancer }{ }^{67} \text {, breast cancer bone metastases }{ }^{22} \text {, ovarian } \\
\text { cervical }^{74} \text {, bone tumour and lung metastasis of osteosarcoma }\end{array}$ \\
\hline LVR01 & AroC & Spinal cord glioma ${ }^{82}$, B-cell lymphoma ${ }^{83}$ \\
\hline CVD915 & GuaBA & T-cell lymphoma ${ }^{84}$ \\
\hline
\end{tabular}




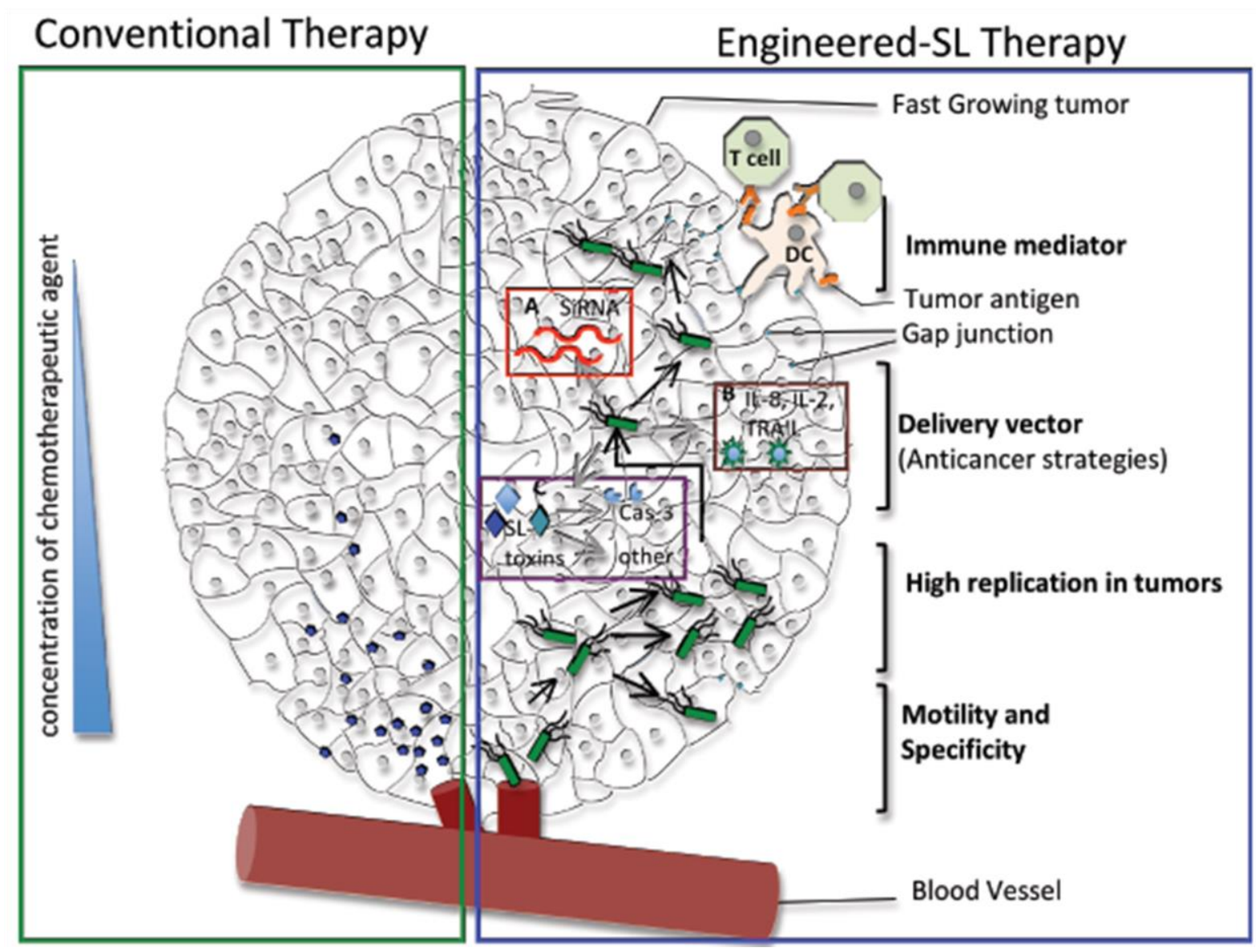

Figure 2: Advantages offered by $S$. Typhimurium (SL) as a cancer therapeutic agent.

(a):gene silencing using siRNA (b):tumour sensitizing (c) tumour regression by caspase-3 mediated mechanisms activated by toxins.

Source: Wall DM, Srikanth CV, McCormick BA. Targeting tumours with Salmonella Typhimurium-potential for therapy. Oncotarget, 2010; 1(8):721-728. doi:https:// doi.org/10.18632/oncotarget.101208

As shown in Figure 2, conventional therapy is indicated in the green box showing the inability of the chemotherapeutic agent to penetrate deep into the tumour. The advantages offered by Salmonella sp. are indicated in the blue box showing its motility, specificity, high replication in tumour cells, formation of gap junctions between adjacent cells and as a delivery vector.

\section{$S$. Typhimurium as a gene delivery vector}

$S$. Typhimurium has been applied as a vector for gene-delivery and other numerous compounds including cytotoxic agents, green fluorescent protein for targeting and visualizing tumours, deoxyribonucleic acid (DNA) used for gene therapy, and small interfering ribonucleic acids (siRNA) to target expression of fundamental proteins within tumours that can be delivered by bacteria to a tumour site. ${ }^{85}$ Multiple $S$. Typhimurium strains notably VNP20009, A1-R, and CRC2631, are being developed for targeted delivery of chemotherapeutic agents. The therapeutic potential of $S$. Typhimurium as a means of delivery has also been evaluated by its ability to easily penetrate and destroy tumour cells. Several promoters of $S$. Typhimurium that preferentially respond to the anaerobic environment of the tumour have been identified ${ }^{86,87}$ and explored as delivery vehicles ${ }^{88}$ for novel anticancer molecules such as Cp53 peptide and L-asparaginase ${ }^{49}$ 
through genetic engineering of the organism, which has resulted in tumour regression. ${ }^{6}$ The use of murine carcinoma cell lines showed that a recombinant strain of attenuated $S$. Typhimurium expressed a cytotoxin, encoded by the gene LIGHT, which had anti-tumour activity without any substantial toxicity. ${ }^{89}$

\section{Combination therapy with $S$. Typhimurium}

Salmonella mediated tumour immunotherapy is no longer focused on using only the organism as a means of battling cancer. Combination of Salmonella with other therapeutic options and strategies with anticancer potential are being explored in quite a number of studies ${ }^{19,90}$ Salmonella was explored as part of a synergistic therapy with chemotherapy using cisplatin, and also as standalone immunotherapy for tumours in mice. ${ }^{91}$ The authors concluded that the synergy of Salmonella and cisplatin exerts more additive therapeutic effects in delaying tumour growth and prolonging the survival of the tumour-bearing mice. Salmonella's ability to suppress the expression of multidrug resistant peptides in tumours has encouraged researchers to utilize several cancer chemotherapeutic agents such as cisplatin or 5-Fluorouracil in combination with Salmonella for treatment. ${ }^{58,92}$ Salmonella also enhances the response to chemotherapeutic agents by increasing the passage of these drugs between neighbouring tumour cells. Combination of Salmonella therapy and cyclophosphamide for treatment improves tumour regression and substantially decreases tumour microvascularization in the melanoma model. ${ }^{93}$ Effect of chemotherapy using CHOP (cyclophosphamide, doxorubicin, vincristine, and prednisone) in B-cell non-Hodgkin lymphoma bearing mice was enhanced as a result of pre-treatment with Salmonella. ${ }^{94}$ Pre-treatment with chemotherapy in the mouse model prior to Salmonella treatment showed enhanced natural killer cell cytotoxic activity and a significantly higher lymphoma-specific humoral and cellular immune responses compared to treatment using Salmonella alone or the chemotherapy alone. ${ }^{94}$

The Salmonella strain VNP20009 was used in combination with photothermal therapy using carbon-based nanomaterial (polydopamine) by coating the strain with polydopamine, designated as pDA-VNP, to function as photothermal agent. ${ }^{95}$ It was discovered that the targeting ability of Salmonella successfully delivered polydopamine to the tumour site and that near-infrared irradiation caused substantial increase in temperature in the tumour site enough to induce tumour cell decay. In vitro cytotoxicity showed significantly lower viable B16F10 cells in pDA-VNP postirradiation group in comparison with VNP20009 alone. ${ }^{96}$

\section{Conclusion}

Bacteria mediated cancer therapy has made major strides in the past decades and is now viewed as a tangible alternative for future cancer therapy. Infection with attenuated $S$. Typhimurium promotes the elimination of tumour cells via intrinsic mechanisms that induce an oncolytic effect on the tumour cell while simultaneously promoting antitumour innate and adaptive immune responses. $S$. Typhimurium as a live attenuated bacterial vector is currently considered to have great potential in the field of cancer immunotherapy but despite the encouraging results from preclinical studies, the approach is yet to be proven successful in clinical practice. Salmonella is an important antitumour agent owing to its tumour-targeting potential, antitumour capability and its capacity to deliver therapeutic genes. 
The potential for bacterial mediated therapy seems infinite but some fundamental issues such as clearance of therapeutic microbes by the host immune system, maintenance of genetic stability in microbes determinants of the susceptibility or resistance of specific cancer types to microbial therapy need to be tackled before this kind of therapy moves into routine clinical practice.

\section{Conflict of interest}

There are no conflicts of interest

\section{Reference}

1. Russell RCG, Williams NS, Bulstrode CJK. Bailey \& Love's short practice of surgery. Arnold. 2000.

2. World Health Organisation (WHO). Latest global cancer data: Cancer burden rises to 18.1 million new cases and 9.6 million cancer death in 2018. Press Release No 263, 12 September, 2018. Retrieved from: $w w w . i a r c . f r$

3. Patyar S, Joshi R, Prasad DS, et al. Bacteria in cancer therapy: a novel experimental strategy. $J$ Biomed Sci, 2010; 17:21-30. doi: http://doi.org/10.1186/1423-0127-17-21

4. Saga K, Kaneda Y. Virosome presents multimodel tumor therapy without viral replication. Biomed Res Int, 2013; 764706 doi: http://doi.org/10.1155/2013/764706

5. Ryan RM, Green J, Lewis CE. Use of bacteria in anticancer therapies. Bioessays. 2006; 28:84-94. doi: http://dx.doi.org/10.1002/bies.20336

6. Nguyen VH, Min JJ. Salmonella-mediated cancer therapy: roles and potential. Nucl Med Mol Imag, 2010; 51:118-126 doi: http://.doi.org/10.1007/s13139-016-0415-z

7. Song S, Vuai1 MS, Zhong M. The role of bacteria in cancer therapy-enemies in the past, but allies at present. Infect Agent Cancer. 2018; 13:9-12 doi: http:// doi.org/10.1186/s13027-018-0180-y

8. McCarthy EF. The toxins of William B. Coley and the treatment of bone and soft-tissue sarcomas. Iowa Orthop J, 2006; 26:154-158. No doi

9. Stritzker J, Weibel S, Seubert C, et al. Enterobacterial tumor colonization in mice depends on bacterial metabolism and macrophages but is independent of chemotaxis and motility. Int J Med Microbiol 2010; 300(7):449-456 doi: http://dx.doi.org/10.1016/j.ijmm.2010.02.004

10. Hernández-Luna MA, Munoz-Lopez P, Luria-Perez R, et al. Infection by Salmonella enterica promotes or demotes tumour development. Salmonella - a re-emerging pathogen 2018; 54-71

doi: http://doi.org/10.5772/intechopen.75481

11. Chavez-Navarro H, Hernández-Cueto DD, Vilchis-Estrada A, et al. Salmonella enterica: An ally in the therapy of cancer. Bol Med Hosp Infant Mex. 2015; 72(1):15-25

doi: http:// doi.org/10.1016/j.bmhimx.2015.02.005

12. Ganai S, Arenas RB, Sauer JP, et al. In tumours Salmonella migrate away from vasculature toward the transition zone and induce apoptosis. Cancer Gene Ther 2011; 18:457-466

doi: http:// doi.org/10.1038/cgt.2011.10

13. Nallar SC, Xu DQ, Kalvakolanu DV. Bacteria and genetically modified bacteria as cancer therapeutics: current advances and challenges. Cytokine 2017; 89:160-172

doi: http:// doi.org/10.1016/j.cyto.2016.01.002

14. Wall DM, Srikanth CV, McCormick BA. Targeting tumours with Salmonella Typhimurium potential for therapy. Oncotarget 2010; 1(8):721-728

doi: http://doi.org/10.18632/oncotarget.101208

15. Wei MQ, Ellem KAO, Dunn P, et al. Facultative or obligate anaerobic bacteria have the potential for multimodality therapy of solid tumours. Euro J Cancer 2007; 43:490-496 doi: http:// doi.org/10.1016/j.ejca.2006.10.005

16. Felgner S, Kocijancic D, Frahm M, et al. Optimizing Salmonella enterica serovar Typhimurium for bacteria mediated tumor therapy. Gut Microbes 2016; 7(2):171-177 
doi: https://doi.org/10.1155/2016/8451728.

17. Wang L, Wang C, Jia Y, et al. Resveratrol increases anti-proliferative activity of Bestatin through downregulating P-Glycoprotein expression via inhibiting PI3K/Akt/mTOR pathway in K562/ADR cells. J Cell Biochem 2016; 117:1233-1239 doi: http:// doi.org/10.1002/jcb.25407

18. Lawrenz HM. Part II: Breakthroughs in tumor-seeking bacteria. Biotechniques. 2018; 64:9-10. doi: http://doi.org/10.2144/000114625

19. Chang WW, Lee CH. Salmonella as an innovative therapeutic antitumor agent. Int J Mol Sci 2014; 51:14546-14554 doi: http:// doi.org/10.3390/ijms150814546

20. Moreno M, Kramer MG, Yim L, Chabalgoity JA. Salmonella as live Trojan horse for vaccine development and cancer gene therapy. Curr Gene Ther, 2010; 10(1):56-76 doi: http://doi.org/10.2174/156652310790945566

21. Pawelek JM, Low KB, Bermudes D. Tumor-targeted Salmonella as a novel anticancer vector. Cancer Res. 1997; 57(20):4537-4544. PMID: 9377566

22. Zhang Y, Miwa S, Zhang N, et al. Tumor-targeting Salmonella Typhimurium A1-R arrests growth of breast-cancer brain metastasis. Oncotarget, 2015; 6:2615-2622.

doi: http://doi.org/10.18632/oncotarget.2811

23. Lee $\mathrm{CH}$. Engineering bacteria toward tumor targeting for tumor treatment: Current state and perspectives. Appl Microbiol Biotechnol 2012; 93:517-523 doi: http://doi.org/10.1007/s00253011-3695-3

24. Forbes NS. Engineering the perfect (bacterial) cancer therapy. Nat Rev Cancer 2010; 10(11):785794 doi: https:// doi.org/10.1038/nrc2934.

25. Hernandez MA, Luria PR, Huerta YS. Therapeutic intervention alternatives in cancer, using attenuated live bacterial vectors: Salmonella enterica as a carrier of heterologous molecules. Rev Invest Clín, 2013; 65(1):65-73. PMID:23745445

26. Kasinskas RW, Forbes NS. Salmonella Typhimurium lacking ribose chemoreceptors localize in tumor quiescence and induce apoptosis. Cancer Res 2007; 67:3201-3209

doi: https:// doi.org/10.1158/0008-5472.CAN-06-2618

27. Cheng M, Bhujwalla ZM, Glunde K. Targeting phospholipid metabolism in cancer. Front Oncol. 2016; 6:266-272 doi:https://doi.org/10.3389/fonc.2016.00266

28. Bovell AM, Warncke K. The structural model of Salmonella Typhimurium ethanolamine ammonialyase directs a rational approach to the assembly of the functional [(EutBEutC)(2)](3) oligomer from isolated subunits. Biochemistry. 2013; 52(8):1419-1428

doi: https://doi.org/10.1021/bi301651n

29. Silva-Valenzuela CA, Desai PT, Molina-Quiroz RC, et al. Solid tumours provide niche-specific conditions that lead to preferential growth of Salmonella. Oncotarget, 2016; 7(23):35169-35180. doi: https:// doi.org/10.18632/oncotarget. 9071.

30. St Jean AT, Zhang M, Forbes NS. Bacterial therapies: completing the cancer treatment toolbox. Curr Opin Biotechol 2008; 19:511-517 doi: https://doi.org/10.1016/j.copbio.2008.08.04

31. Martinez UE, Peiris-Pages M, Pestell RG, et al. Cancer metabolism: A therapeutic perspective. Nat Rev Clin Oncol. 2017; 14(2):113 doi: https://doi.org/10.1038/nrclinonc.2017.1

32. Crull K, Weiss S. Antibiotic control of tumor-colonizing Salmonella enterica serovar Typhimurium. Exp Biol Med (Maywood). 2011; 236:1282-1290.

doi: https://doi.org/10.1258/ebm.2011.011111

33. Hernandez LD, Pypaert M, Flavell RA, et al. A Salmonella protein causes macrophage cell death by inducing autophagy. J Cell Biol. 2003; 163:1123-1131

doi: https:// doi.org/10.1083/jcb.200309161

34. Gomes LC, Dikic I. Autophagy in antimicrobial immunity. Mol Cell. 2014; 54:224-233

doi: https:// doi.org/10.1016/j.molcel.2014.03.009

35. Pangilinan CR, Che-Hsin L. Salmonella-based targeted cancer therapy: updates on a promising and innovative tumor immunotherapeutic strategy. Biomed 2019; 7:36-50 
doi: https://doi.org/10.3390/biomedicines7020036

36. Lee CH, Lin ST, Liu JJ, et al. Salmonella induce autophagy in melanoma by the down-regulation of AKT/mTOR pathway. Gene Ther 2014; 21:309-316 doi: https://doi.org/10.1038/gt.2013.86

37. Boise LH, Collins CM. Salmonella-induced cell death: Apoptosis, necrosis or programmed cell death. Trends Microbiol. 2001; 9:64-67 doi: https://doi.org/10.1016/s0966-842x(00)01937-5

38. Kaimala S, Mohamed YA, Nader N, et al. Salmonella-mediated tumor regression involves targeting of tumor myeloid suppressor cells causing a shift to M1-like phenotype and reduction in suppressive capacity. Cancer Immunol, Immunother 2014; 63(6):587-599

doi: https:// doi.org/10.1007/s00262-014-1543-x

39. Lukasiewicz K, Fol M. Microorganisms in the treatment of cancer: advantages and limitations. $J$ Immunol Res 2018; 2397808 doi: https://doi.org/10.1155/2018/2397808

40. Spranger S, Gajewski, TF. Mechanisms of tumor cell-intrinsic immune evasion. Annual Rev Cancer Biol 2018; 2:213-228 doi: https://doi.org/10.1146/annurev-cancerbio-030617-050606

41. Lee $\mathrm{CH}$, Hsieh JL, Wu CL, et al. T cell augments the antitumor activity of tumor targeting Salmonella. Appl Microbiol Biotechnol, 2011a; 90(4):1381-1388 doi: https://doi.org/10.1007/s00253-011-3180-z

42. Torres W, Lameda V, Olivar LC, et al. Bacteria in cancer therapy; beyond immunostimulation. $J$ Cancer Meta Treat 2018; 4:4-10. doi: 10.20517/2394-4722.2017.49

43. Tam JW, Kullas AL, Mena P, et al. CD11b+ Ly6Chi Ly6G- immature myeloid cells recruited in response to Salmonella enterica serovar Typhimurium infection exhibit protective and immunosuppressive properties, Infect Immun 2014; 82(6): 2606-2614

doi: https://doi.org/10.1128/IAI.01590-13

44. Grille S, Moreno M, Bascuas T, et al. Salmonella enterica serovar Typhimurium immunotherapy for B-cell lymphoma induces broad anti-tumour immunity with therapeutic effect. Immunology. 2014; 143(3):428-437 doi: https://doi.org/10.1111/imm.12320

45. Barak YF, Schreiber F, Thorne SH, et al. Role of nitric oxide in Salmonella Typhimurium-mediated cancer cell killing. BMC Cancer, 2010; 10(1):146-152

doi: https:// doi.org/10.1186/1471-2407-10-146

46. Lee $\mathrm{CH}$, Hsieh JL, Wu CL, et al. B cells are required for tumor-targeting Salmonella in host.

Appl Microbiol Biotechnol. 2011b; 92(6):1251-1260

doi: https://dx.doi.org/10.1007/s00253-011-3386-0

47. Kocijancic D, Leschner S, Felgner S, et al. Therapeutic benefit of Salmonella attributed to LPS and TNF- $\alpha$ is exhaustible and dictated by tumor susceptibility. Oncotarget, 2017; 8:36492-36508 doi: https://doi.org/10.18632/oncotarget.16906

48. Lee CH, Wu CL, Shiau AL. Toll-like receptor 4 mediates an antitumor host response induced by Salmonella Choleraesuis. Clin Cancer Res 2008; 14:1905-1912

doi: https://doi.org/10.1158/1078-0432-CCR-07-2050

49. Kim, J.E., Phan, T.X., Nguyen, V.H., et al. Salmonella typhimurium suppresses tumor growth via the pro-inflammatory cytokine interleukin-1beta. Theranos 2015; 5:1328-1342

doi: https:// doi.org/10.7150/thno.11432.

50. Leschner S, Westphal K, Dietrich N, et al. Tumor invasion of Salmonella enterica serovar Typhimurium is accompanied by strong hemorrhage promoted by TNF- $\alpha$, PLOS One, 2009; 4(8):e6692 doi: https:// doi.org/10.1371/journal.pone.0006692.

51. Chirullo B, Ammendola S, Leonardi L, et al. Attenuated mutant strain of Salmonella Typhimurium lacking the ZnuABC transporter contrasts tumor growth promoting anti-cancer immune response. Oncotarget 2015; 6(19):17648-17660 doi: https://doi.org/10.18632/oncotarget.3893

52. Maybeno M, Redeker A, Welten SP, et al. Polyfunctional CD4+ T cell responses to immunodominant epitopes correlate with disease activity of virulent Salmonella. PLoS One 2012; 7:e43481 doi: https://doi.org/10.1371/journal.pone.0043481 
53. Saccheri F, Pozzi C, Avogadri F, et al. Bacteria-induced gap junctions in tumours favor antigen cross-presentation and antitumor immunity. Sci Transl Med 2010; 2:44-57

doi: https://doi.org/10.1126/scitranslmed.3000739

54. Linley AJ, Ahmad M, Rees RC. Tumour-associated antigens: considerations for their use in tumour immunotherapy. Int J Hematol. 2011; 93(3):263-273

doi: https://doi.org/10.1007/s12185-011-0783-1

55. Santos RL, Baumler AJ. Cell tropism of Salmonella enterica. Int J Med Microbiol 2004; 294(4):225-233 doi: https://doi.org/10.1016/j.ijmm.2004.06.029

56. Avogadri F, Martinoli C, Petrovska L, et al. Cancer immunotherapy based on killing of Salmonella-infected tumor cells, Cancer Res, 2005; 65(9):3920-3927

doi: https://doi.org/10.1158/0008-5472.Can-04-3002

57. Zhao M, Yang M, Li XM, et al. Tumor-targeting bacterial therapy with amino acid auxotrophs of GFPexpressing Salmonella Typhimurium. Proct Natl Acad Sci U S A 2005; 102:755-760

doi: https://doi.org/10.1073/pnas.0408422102

58. Chang WW, Lai CH, Chen MC, et al. Salmonella enhance chemosensitivity in tumor through connexin 43 upregulation. Int J Cancer 2013; 133:1926-1935

doi: https://doi.org/10.1002/ijc.28155

59. Stern C, Kasnitz N, Kocijancic D, et al. Induction of CD4(+) and CD8(+) anti-tumor effector T cell responses by bacteria mediated tumor therapy. Int J Cancer 2015; 137:2019-2028

doi: https://doi.org/10.1002/ijc.29567

60. Lee $\mathrm{CH}, \mathrm{Wu} \mathrm{CL}$, Chen $\mathrm{SH}$, et al. Humoral immune responses inhibit the antitumor activities mediated by Salmonella enterica serovar Choleraesuis. J Immunother 2009; 32:376-388

doi: https://doi.org/10.1097/CJI.0b013e31819d4ebc

61. Palffy R, Gardlik R, Hodosy J, et al. Bacteria in gene therapy: Bactofection versus alternative gene therapy. Gene Ther 2006; 13:101-105 doi: https://doi.org/10.1038/sj.gt.3302635

62. Felgner S, Kocijancic D, Frahm M, et al. Engineered Salmonella enterica serovar Typhimurium overcomes limitations of anti-bacterial immunity in bacteria-mediated tumor therapy, Oncoimmunology 2018;7:2,e1382791 doi: https:// doi.org/10.1080/2162402X.2017.1382791

63. Jazeela K, Chakraborty A, Karunasagar I, et al. Nontyphoidal Salmonella: a potential anticancer agent. J Appl Microbiol 2019; 338-351 doi: https://doi.org/10.1111/jam.14297

64. Din MO, Danino T, Prindle A, et al. Synchronized cycles of bacterial lysis for in vivo delivery. Nature 2016; 536:81-85 doi: https://dx.doi.org/10.1038/nature18930

65. Dang LH, Bettegowda C, Huso DL, et al. Combination bacteriolytic therapy for the treatment of experimental tumours. Proct Natl Acad Sci USA. 2001; 98:15155-15160

doi: https:// doi.org/10.1073/pnas.251543698

66. King I, Itterson M, Bermudes D. Tumor-targeted Salmonella Typhimurium overexpressing cytosine deaminase: a novel, tumor-selective therapy. Methods Mol Biol. 2009; 542:649-659

doi: https://doi.org/10.1007/978-1-59745-561-9_33

67. Zhao M, Yang M, Ma H, Targeted therapy with a Salmonella Typhimurium leucine-arginine auxotroph cures orthotopic human breast tumour in nude mice. Cancer Res 2006; 66:7647-7652 doi: https://doi.org/10.1158/0008-5472.

68. Zhao M, Geller J, Ma H, et al. Monotherapy with a tumor-targeting mutant of Salmonella Typhimurium cures orthotopic metastatic mouse models of human prostate cancer. Proc Natl Acad Sci USA 2007; 104:10170-10174 doi: https:// doi.org/10.1073/pnas.0703867104.

69. Liu T, Chopra AK. An enteric pathogen Salmonella enterica serovar Typhimurium suppresses tumor growth by downregulating CD44high and CD4T regulatory (Treg) cell expression in mice: the critical role of lipopolysaccharide and Braun lipoprotein in modulating tumor growth. Cancer Gene Ther. 2010; 17(2):97-108 doi: https://doi.org/10.1038/cgt.2009.58. 
70. Hiroshima Y, Zhao M, Zhang Y, et al. Comparison of efficacy of Salmonella Typhimurium A1-R and chemotherapy on stem-like and non-stem human pancreatic cancer cells. Cell Cycle 2013; 12:2774-2780. doi: https://doi.org/10.4161/cc.25872

71. Yam C, Zhao M, Hayashi K, et al. Monotherapy with a tumor-targeting mutant of S. Typhimurium inhibits liver metastasis in a mouse model of pancreatic cancer. J Surg Res 2010; 164:248-255 doi: https://doi.org/10.1016/j.jss.2009.02.023

72. Matsumoto Y, Miwa S, Zhang Y, et al. Intraperitoneal administration of tumor-targeting Salmonella Typhimurium A1-R inhibits disseminated human ovarian cancer and extends survival in nude mice. Oncotarget. 2015; 6:11369-11377 doi: https://doi.org/10.18632/oncotarget.3607

73. Yano S, Zhang Y, Zhao M, et al. Tumor-targeting Salmonella Typhimurium A1-R decoys quiescent cancer cells to cycle as visualized by FUCCI imaging and become sensitive to chemotherapy. Cell Cycle 2014; 13:3958-3963 doi: https:// doi.org/10.4161/15384101.2014.964115

74. Hiroshima, Y, Zhang Y, Zhao M, et al. Tumor-targeting Salmonella Typhimurium A1-R in combination with Trastuzumab eradicates HER-2-positive cervical cancer cells in patient-derived mouse models. PLoS One 2015; 10:e120358 doi: https:// doi.org/10.1371/journal.pone.0120358.

75. Hayashi K, Zhao M, Yamauchi K, et al. Cancer metastasis directly eradicated by targeted therapy with a modified Salmonella Typhimurium. J Cell Biochem. 2009; 106:992-998

doi: https:// doi.org/10.1002/jcb.22078

76. Kimura H, Zhang L, Zhao M, et al. Targeted therapy of spinal cord glioma with a geneticallymodified Salmonella Typhimurium. Cell Prolif. 2010; 43(1):41-48

doi: https:// doi.org/10.1111/j.1365-2184-2009.00652.x

77. Momiyama M, Zhao M, Kimura H, et al. Inhibition and eradication of human glioma with tumortargeting Salmonella Typhimurium in an orthotopic nude-mouse model. Cell Cycle, 2012; 11:628632 doi: https://doi.org/10.4161/cc.11.3.19116

78. Crull K, Bumann D, Weiss S. Influence of infection route and virulence factors on colonization of solid tumors by Salmonella enterica serovar Typhimurium. FEMS Immunol Med Micriobiol. 2011; 62(1):75-83 doi: https://doi.org/10.1111/j.1574-695X.2011.00790.x

79. Eisenstein TK, Bushnell B, Meissler JJ, et al. Immunotherapy of a plasmacytoma with attenuated Salmonella. Med Oncol. 1995; 12(2):103-108 doi: https://doi.org/10.1007/BF01676710

80. Toso JF, Gill VJ, Hwu P, et al. Phase I study of the intravenous administration of attenuated Salmonella typhimurium to patients with metastatic melanoma. J Clinc Oncol. 2002; 20(1):142152 doi: https://doi.org/10.1200/JCO.2002.20.1.1.142

81. Choe E, Kazmierczak RA, Eisenstark A. Phenotypic evolution of therapeutic Salmonella enterica serovar Typhimurium after invasion of TRAMP mouse prostate tumor. MBio. 2014; 5(4):e01182e01114 doi: https://doi.org/10.1128/mBio.01182-14.

82. Kimura H, Zhang L, Zhao M, et al. Targeted therapy of spinal cord glioma with a genetically modified Salmonella Typhimurium. Cell Prolif. 2010; 43(1):41-48 doi: https://doi.org/10.1111/j.1365-2184.2009.00652.x

83. Grille S, Moreno M, Brugnini A, et al. A therapeutic vaccine using Salmonella-modified tumor cells combined with interleukin-2 induces enhanced antitumor immunity in B-cell lymphoma. Leukemia Res. 2013; 37(3):341-348 doi: https://doi.org/10.1016/j.leukres.2012.10.003

84. Vendrell A, Gravisaco MJ, Goin JC et al. Therapeutic effects of Salmonella Typhi in a mouse model of T-cell lymphoma. J Immunother. 2013; 36(3):171-180 doi: https://doi.org/10.1097/CJI.ob013e3182886d95

85. Basu D, Herlyn M. Salmonella Typhimurium as a novel RNA interference vector for cancer gene therapy. Cancer Biol Ther. 2008; 7:151-152. PMID:18347415

86. Arrach N, Zhao M, Porwollik S, et al. Salmonella promoters preferentially activated inside tumors. Cancer Res 2008; 68:4827-4832 doi: https:// doi.org/10.1158/0008-5472.CAN.09.4005

87. Leschner S, Deyneko IV, Lienenklaus S, et al. Identification of tumor-specific Salmonella Typhimurium promoters and their regulatory logic. Nucleic Acids Res 2012; 40:2984-2994 
doi: https://doi.org/10.1093/nar/gkr1041

88. Camacho EM, Mesa-Pereira B, Medina C, et al. Engineering Salmonella as intracellular factory for effective killing of tumour cells. Sci Rep 2016; 6:30591 doi: https://doi.org/10.1038/srep30591

89. Loeffler M, Le'Negrate G, Krajewska M, et al. Salmonella Typhimurium engineered to produce CCL21 inhibit tumor growth. Cancer Immunol Immunother. 2009; 58(5):769-775

doi: https://doi.org/10.1007/s00262-008-0555-9

90. Nguyen VH, Min JJ. Salmonella-Mediated Cancer Therapy: Roles and Potential. Nucl Med Mol Imag, 2017; 51:118-126. doi: https://doi.org/10.1007/s13139-016-0415-z

91. Lee CH, Wu CL, Tai YS, et al. Systemic administration of attenuated Salmonella choleraesuis in combination with cisplatin for tumor therapy. Mol Ther. 2005; 11:707-716

doi: https:// doi.org/10.1016/j.ymthe.2005.01.008

92. Yang CJ, Chang WW, Lin ST, et al. Salmonella Overcomes Drug resistance in tumor through Pglycoprotein downregulation. Int J Med Sci 2018; 15:574-579 doi: https://doi.org/10.7150/ijms.23285

93. Jia LJ, Wei DP, Sun QM, et al. Tumor-targeting Salmonella Typhimurium improves cyclophosphamide chemotherapy at maximum tolerated dose and low-dose metronomic regimens in a murine melanoma model. Int J Cancer 2007; 121:666-674

doi: https://doi.org/10.1002/ijc.22688

94. Bascuas T, Moreno M, Grille S, et al. Salmonella immunotherapy improves the outcome of CHOP chemotherapy in non-Hodgkin lymphoma-bearing mice. Front Immunol, 2018; 9:7-12 doi: https:// doi.org/10.3389/fimmu.2018.00007

95. Chen W, Wang Y, Qin M, et al. Bacteria-driven hypoxia targeting for combined biotherapy and photothermal therapy. ACS Nano 2018; 12:5995-6005 doi: https:// doi.org/10.1021/acsnano.8b02235

96. Zhu H, Cheng P, Chen P, Pu K. Recent progress in the development of near-infrared organic photothermal and photodynamic nanotherapeutics. Biomater Sci 2018; 6:746-765

doi: https://doi.org/10.1039/C7BM01210A 